\title{
Properties of the O-type giants and supergiants in 30 Doradus
}

\author{
O. H. Ramírez-Agudelo and VFTS consortium \\ UK ATC, Royal Observatory Edinburgh, Blackford Hill, Edinburgh, EH9 3HJ, UK \\ email: oscar.ramirez@stfc.ac.uk
}

\begin{abstract}
We discuss the stellar and wind properties of 72 presumably single O-type giants, bright giants, and supergiants in the 30 Doradus region. This sample constitutes the largest and most homogeneous sample of such stars ever analyzed and offers the opportunity to test models describing their main-sequence evolution.
\end{abstract}

Keywords. stars: early-type - stars: evolution - stars: fundamental parameters - Magellanic Clouds - Galaxies: star clusters: individual: 30 Doradus

The 30 Doradus (30 Dor) region in the Large Magellanic Cloud (LMC) is one of the best possible laboratory to investigate aspects of the formation and evolution of massive stars. In the framework of the VLT-FLAMES Tarantula Survey (VFTS; Evans et al. 2011), we determine stellar and wind properties of 72 presumably single O-type stars in 30 Dor with luminosity class (LC) III (giants), II (bright giants), and I (supergiants).

We apply an automated fitting method for quantitative spectroscopic analysis of Ostars to determine the following key stellar properties of our sample of stars: the effective temperature, the surface gravity, the mass-loss rate, the helium abundance, the microturbulent velocity, and the projected rotational velocity. Details on the method are given in Ramírez-Agudelo et al. 2017.

Our main findings can be summarized as follows:

- We present an empirical effective temperature versus spectral subtype calibration for the stars with LC III and LCI. The calibration for the LC III shows a $+1 \mathrm{kK}$ offset compared to similar Galactic calibrations.

- According to the spectroscopic and classical Hertzsprung-Russell diagrams our sample of $\mathrm{O}$ stars occupy the region predicted for the core hydrogen-burning phase. Late LC III and LC II stars occupy the region where O9.5-9.7 V stars are expected, but where few morphologically-classified dwarfs are seen. This behavior may reflect an intricacy in the luminosity classification at late $\mathrm{O}$ spectral subtype.

- The surface helium abundances of our sample stars are generally in agreement with the initial composition of the LMC, but five stars display enriched helium abundances. These He-enriched stars have moderate rotational velocities $\left(<200 \mathrm{~km} \mathrm{~s}^{-1}\right)$, and hence do not agree with predictions of rotational mixing in main-sequence stars. They may reveal other physics not including in the models, such as binary-interaction effects.

- Adopting theoretical results for the wind velocity law, for stars brighter than $10^{5} L_{\odot}$ the measured (unclumped) mass-loss rates can be brought in agreement with predictions if the clump filling factor in the $\mathrm{H} \alpha$ and He II $\lambda 4686$-forming region were $\sim 1 / 8$ to $1 / 6$.

\section{References}

Evans et al., 2011, A\&A, 530, A108

Ramírez-Agudelo et al., 2017, A\&A, 600, A81 